\title{
Post-partum family planning in Burkina Faso (Yam Daabo): a two group, multi-intervention, single-blinded, cluster-randomised controlled trial
}

Nguyen Toan Tran, Armando Seuc, Abou Coulibaly, Sihem Landoulsi, Tieba Millogo, Fatou Sissoko, Wambi Maurice E Yameogo, Souleymane Zan, Asa Cuzin-Kihl, James Kiarie, Mary Eluned Gaffield, Blandine Thieba, Seni Kouanda

\section{Summary}

Background Post-partum family planning services can prevent maternal and child morbidity and mortality in lowresource settings. We assessed the effect of a family planning intervention package on modern contraceptive use at 12 months post partum in predominantly rural Burkina Faso.

Methods Yam Daabo was a two group, multi-intervention, single-blinded, cluster randomised controlled trial. Primary health-care centres were randomly allocated to intervention or control clusters in a 1:1 ratio with only data analysts masked to the allocation assignment. Interventions comprised refresher training for the provider, a counselling tool, supportive supervision, availability of contraceptive services 7 days a week, client appointment cards, and invitation letters for partners. The primary outcome was modern contraceptive prevalence at 12 months, and secondary outcomes were modern contraceptive prevalence at 6 weeks and 6 months post partum. Analysis was by modified intention to treat. Prevalence ratios were adjusted for cluster effects and baseline characteristics. This study was registered with the Pan-African Clinical Trials Registry (PACTR201609001784334).

Findings From July 27-Oct 17, 2016, eight clinics were randomised and 571 women were enrolled and allocated: 286 to four intervention clusters and 285 to four control clusters. Of these, 523 completed the 12-month study exit interview (260 in the intervention group, 263 in the control group) and 523 were included in the intention-to-treat analysis. At 12 months, modern contraceptive prevalence was $55 \%$ among women who received the package and $29 \%$ among those who received routine care in control clusters (adjusted prevalence ratio 1.79, 95\% CI 1·30-2.47). Significant differences in modern contraceptive prevalence were also seen between intervention and control groups at 6 weeks $(42 \%$ and $10 \%$, respectively; adjusted prevalence ratio $3 \cdot 88,95 \%$ CI $1 \cdot 46-10 \cdot 35)$ and 6 months $(59 \%$ and $24 \%$, respectively; $2 \cdot 31,1 \cdot 44-3 \cdot 71)$.

Interpretation A package of six low-technology interventions, aimed at strengthening existing primary health-care services and enhancing demand for these services, can effectively increase modern contraceptive use for up to a year post partum in rural settings in Burkina Faso and has the potential to be suitable in similar settings in this country and others.

Funding Government of France.

Copyright (C) 2019 The Author(s). Published by Elsevier Ltd. This is an Open Access article under the CC BY 4.0 license.

\section{Introduction}

Spacing birth-to-pregnancy intervals by at least 2 years and birth-to-birth intervals by at least 3 years could prevent more than $30 \%$ of maternal and $10 \%$ of infant mortality. ${ }^{1}$ WHO defines post-partum family planning (PPFP) as the prevention of unwanted pregnancies and closely-spaced pregnancies during the first 12 months after delivery when pregnancy poses the greatest risk to mother and baby. Hence, the aim of PPFP services is to help women and couples to decide on the method they want to use, to initiate that method, and to continue use for ideally 2 years or longer, depending on their reproductive intentions. ${ }^{2}$

Birth-to-pregnancy intervals in $50 \%$ or more of pregnancies in low-income and middle-income countries are too short-at less than 23 months. $^{3}$ The unmet need for family planning in the post-partum period of 12 months is high and far exceeds the unmet need for family planning of women who are not in the postpartum period. Depending on the definitions of unmet need for PPFP and according to estimates derived from Demographic and Health Survey data analysis from 57 countries, including 32 in sub-Saharan Africa, the proportion of post-partum women who want to postpone another child for at least 2 years but are not using contraception ranges from $32 \%$ (risk assumed to resume at the end of amenorrhoea) to $62 \%$ (risk assumed to resume soon after birth and before the sixth week post partum [given that pregnancy can occur even before menses return]). ${ }^{4}$ With the definition that the risk is assumed to resume soon after birth and before the sixth week post partum, the unmet need the year after birth
Lancet Glob Health 2019; 7: e1109-17

See Comment page e996 Department of Reproductive Health and Research, World Health Organization, Geneva, Switzerland (NTTran DrMed, A Seuc PhD, S Landoulsi MSc A Cuzin-Kihl MSc, J Kiarie MD, M E Gaffield PhD); Australian Centre for Public and Population Health Research, Faculty of Health, University of Technology, Sydney, Australia (NTTran); Institut de Recherche en Sciences de la Santé and Institut Africain de Santé Publique, Ouagadougou, Burkina Faso (A Coulibaly MD, T Millogo MD, F Sissoko MSc, W M EYameofo MSC, S Kouanda PhD); World Health Organization Country Office in Burkina Faso, Ouagadougou, Burkina Faso (S Zan MD); and Unité de Formation et de Recherche en Sciences de la Santé, Ouagadougou University, Ouagadougou, Burkina Faso (B Thieba MD)

Correspondence to: Dr Nguyen Toan Tran Department of Reproductive Health and Research, World Health Organization, Avenue Appia 20, 1211 Geneva 27, Switzerland nguyen-toan.tran@unige.ch 
Research in context

\section{Evidence before this study}

On Aug 11, 2018, we searched PubMed using the term "randomized controlled trial" combined with "postpartum" and "family planning" or "contraception" for publications in English focusing on trials in developing countries. We identified five trials since the last systematic review, which was published in 2016. All five trials were in Africa (Burkina Faso, Egypt, Kenya, Rwanda, and Uganda) and investigated a specific intervention. The urban-based Burkina Faso study showed that involving male partners of pregnant women in maternity care through group sessions targeting only men and couple counselling sessions before and after childbirth resulted in marginal differences in the use of any contraceptives at 8 months post partum between the intervention group (71\%) and control group (64\%), with a risk ratio of $1 \cdot 10$ ( $95 \% \mathrm{Cl} 1 \cdot 02-1 \cdot 20)$. The university hospital-based Egyptian study, which recruited pregnant women who were planning to space their next pregnancy and breastfeed for at least a year, confirmed previous findings: in-advance counselling and provision of emergency contraceptive pills had a significant effect on the proportion of unplanned pregnancies at 6 months post partum between the emergency contraceptive pill and lactational amenorrhoea group (0.4\%) and lactational amenorrhoea only group (5.3\%) $(p=0.0001)$. In Kenya, the use of mobile phone short message service sent weekly to urban women in the antenatal and post-partum period did not show a difference in contraceptive use at 10,16, or 24 weeks post partum. In Rwanda, post-partum family planning (PPFP) services integrated into immunisation clinics appeared to be feasible, acceptable, and effective in increasing modern contraceptive use (49\% of women at baseline against $57 \%$ at endline in the intervention group), although it is important to note that these were different groups of women (in the control group, the proportion of women using a modern method was $58 \%$ at baseline and $50 \%$ at endline). The university hospital-based
Ugandan study recruited women just after childbirth who wanted a contraceptive implant upon receiving comprehensive contraceptive counselling. Implant use at 6 months was higher among women who had insertion before hospital discharge (97\%) versus those who chose to delay insertion to 6 weeks post partum $(68 \% ; \mathrm{p}<0 \cdot 001)$.

\section{Added value of this study}

The Yam Daabo study did not investigate a specific programmatic innovation or the promotion of a particular PPFP method-fertility and contraceptive choices were made by the women and couples. This cluster-randomised controlled trial showed that a package of six low-technology interventions, designed in a participatory manner and aimed at strengthening routine antenatal and postnatal services in primary health clinics in predominantly rural settings (ie, refresher training of service providers, regularly scheduled and strengthened supportive supervision of providers, enhanced availability of services 7 days a week, a PPFP counselling tool, appointment cards for women, and invitation letters for partners), doubled the proportion of women using short-acting and long-acting reversible modern contraceptives up to 12 months post partum when compared with women using routine care.

Implications of all the available evidence

On the basis of the growing body of evidence on PPFP, decision makers can opt for different strategies to strengthen contraceptive use depending on health service needs, gaps, and opportunities of specific contexts. These strategies include the Yam Daabo approach, which aimed to reinforce existing routine services through a package of different interventions. Although cost-effectiveness of the package warrants further study, the low technology of the interventions and focus on strengthening existing services at the primary health-care level make it suitable for large-scale implementation in similar settings in Burkina Faso and other countries. was $65 \%$ in east and southern Africa and even reached $75 \%$ in west and central Africa. ${ }^{4}$ In the footsteps of FP2020, a global initiative to reprioritise family planning as a development objective, a PPFP movement was initiated in 2015 to address this high unmet need.

According to the most recent Demographic and Health Survey data available from Burkina Faso (2010), the median duration of breastfeeding is 24 months (0.6 months for exclusive breastfeeding), the median post-partum sexual abstinence is 8 months, and the median duration of amenorrhoea is 12 months. ${ }^{5}$ The median birth interval is 36 months, yet $13 \%$ of babies are born less than 24 months after their previous sibling. Most women (95\%) attend antenatal care and deliver in a health facility (66\%). Postnatal care is offered to $72 \%$ of post-partum women within $48 \mathrm{~h}$ of childbirth, and $81 \%$ of children $12-23$ months receive all recommended vaccines. Despite this high uptake of routine reproductive, maternal, newborn, and child health services that offer frequent contact points to address the contraceptive needs of post-partum women and couples, ${ }^{6}$ the unmet need for family planning among post-partum women is high at $89 \%$ right after birth, $50 \%$ after 6 months of amenorrhoea, and $21 \%$ at the end of amenorrhoea. The unmet need among all married women of reproductive age is also high at 24\%. ${ }^{4}$ In 2015, data from the United Nations showed that maternal mortality remained high in Burkina Faso (371 of 100000 live births), as did the total fertility rate ( 5.4 children per woman).

PPFP is usually designed as an integral part of reproductive, maternal, newborn, and child health services. Although advances in access to these services in many sub-Saharan African countries, including Burkina Faso, have been observed in recent decades, progress is slow regarding effective post-partum contraceptive use. ${ }^{3,8,9}$ The evidence is often weak or 
incomplete in terms of study design and quality, intervention details, or women's perspectives according to various literature reviews. ${ }^{10-12}$ Additionally, knowledge gaps still exist, particularly regarding studies that look at operationally feasible ways to integrate PPFP into existing antenatal care and postnatal care (eg, by meaningfully engaging community stakeholders). ${ }^{2}$ To address the high unmet need for PPFP, the objective of Yam Daabo (ie, "your choice" in Mooré, one of the local languages in Burkina Faso) was to determine the effectiveness of a PPFP intervention package designed applying participatory action research on the use of contraceptive methods during the first year post partum. In anticipation of potential dissemination of the Yam Daabo strategy, the trial took place in rural and urban settings and in two countries (ie, Burkina Faso and the Democratic Republic of Congo); here, we report the results of the Burkina Faso trial.

\section{Methods \\ Study design and participants}

The study was implemented in Passoré province, Northern Region and one of the poorest areas of Burkina Faso, in the rural villages of Arbole, Bagaré, Bouré, Gomponsom, Samba, and Tema, and the urban Sectors of Yako (Secteur 5 and Secteur 6). Further details on Burkina Faso and the study sites are provided in the appendix, pp 2-3.

Yam Daabo was a pragmatic two-group, clusterrandomised, multi-intervention trial done in eight primary health centres, at the level of which randomisation to control and intervention arms occurred (see published protocol). ${ }^{13}$ The cluster randomised controlled trial design was justified by the fact that three of the six components of the PPFP package were not delivered directly to individual participants but only implemented at the facility level. In addition, the cluster design avoided potential contamination between intervention and control groups for the other three individual-based interventions. Participants randomly allocated to the intervention group received the PPFP intervention package whereas individuals randomly allocated to the control group received routine health care.

The study was done in centres offering reproductive, maternal, newborn, and child-health services. Further details of the sampling calculation are provided in the published protocol. ${ }^{13}$ Health centres were eligible if they offered the continuum of antenatal care, delivery, and postnatal care; had at least three modern contraceptive methods available, including a barrier method such as condoms, a short-term method such as pills, a long-term method such as intrauterine devices, and referrals for permanent methods; had no stock-outs of contraceptives during the preceding 6 months; had on average at least 30 deliveries per month; were situated within a $4 \mathrm{~h}$ drive from the research centre; and were willing to participate. All pregnant women attending the health centres for antenatal care were invited to participate in the study. They were eligible if they were in their third pregnancy trimester; their health and pregnancy situation allowed for a birth at the health centre; they had the intention to attend antenatal care, delivery, and postnatal care at the health centre; they were not participating in another study; and they provided informed consent (and assent for minors of $<18$ years old).

\section{Randomisation and masking}

The eight sites were matched in pairs according to the number of monthly deliveries, the ratio of health workers per population in the health zone, and the setting (ie, rural, urban). Within each of the four pairs, we randomly assigned a site to the intervention group or to the control group. This randomisation was done four times (once for each pair). No restriction in the randomisation process was required. All consecutive and eligible participants were included in the clusters. Due to the nature of the interventions, participants, health staff, research assistants assigned to each centre, and the rest of the research team members could not be masked to the cluster assignments. Only data analysts were masked to the allocation assignment (ie, they received no information about the cluster allocation and did not interact with the field team).

Study approval was obtained by the ethics committee in Burkina Faso (reference number: 2016-02-019) and WHO Research Ethics Review Committee, Geneva, Switzerland (Protocol ID RPC757). All adult participants provided written consent. Minors gave their informed assent and their respective parents or guardians their informed consent.

\section{Intervention package}

The rationale for our approach is based on the WHO seminal publication in 2013 of Programmatic strategies for postpartum family planning, and three other systematic reviews of the literature (without meta-analysis) on PPFP interventions in less developed countries published between 2014 and 2016., ${ }^{211,12,14}$ These documents suggest that the following interventions may have a positive effect on post-partum contraceptive uptake: counselling activities during antenatal care; provision of PPFP information, education, and counselling materials before women are discharged home from health facilities, including provision of emergency contraception backup for users of the lactational amenorrhoea method; promotion by community-based counsellors of exclusive breastfeeding practices before 5-6 months post partum; access to contraceptive methods immediately after childbirth, including intrauterine devices; provider competencies in quality counselling and the provision of quality services with a range of readily available products; and longer programmes with several contact points between providers and clients across the continuum of care versus short antenatal interventions. 
Our PPFP intervention package comprised three facilityoriented interventions (ie, refresher training of service providers, regularly scheduled and strengthened supportive supervision of providers, enhanced availability of services 7 days a week), and three individual-based interventions (ie, a PPFP counselling tool, appointment cards for women, and invitation letters for partners). The package was designed through participatory action research and the process and contents are detailed elsewhere. ${ }^{15,16}$ Participants received individual-based interventions during third-trimester antenatal care visits and postnatal care follow-up visits, according to national practice (typically on clinical discharge [24-48 h], at 6 days, 6 weeks, then at months 6 and 9, before the trial exit at month 12 post partum).

The working hypotheses for the selection of the PPFP interventions to be included in the package were as follows. They should strengthen existing antenatal care and postnatal care services by means of so-called lowdose, high-frequency interventions (which we assumed would be more effective than high-dose, low-frequency strategies that promote a specific method over another and restrict services to a narrow time window, such as before home discharge after childbirth, or during the 6-week postnatal care visits dedicated to PPFP); meaningfully engage key actors, including clients and providers, in the design, implementation, and research to better reflect field reality (including limited human resources and clinical capacity), and ensure its feasibility, sustainability, and scalability to other settings; be in line with national health policies; and take into consideration the scarcity of public resources.

The WHO team in Geneva developed the paper-based study case report forms with inputs from the country researchers who tested advanced drafts with an appropriate sample of mock clients from sites included in the formative study phase. Data were collected by trained research assistants, irrespective of whether women attended clinic visits after enrolment. Data entry was performed in Ouagadougou and checked in Geneva by use of OpenClinica (version 3.11).

\section{Outcomes}

The primary outcome was prevalence of modern contraceptive use at 12 months post partum (assessed on the first day of month 12). We amended our original protocol to extend the follow-up period from months 9 to 12 post partum to allow for better comparability with other published research, after the initial funder's research grant deadline was chosen. This extension was approved by Ethics Committees at WHO Headquarter and country level. We also report two secondary outcomes: prevalence of modern contraceptive use at 6 weeks (assessed at 45 days), which coincides with the PPFP-dedicated visit as per national recommendations, and prevalence of modern contraceptive use at 6 months (assessed on the first day of month 6), which corresponds to the latest point when transition from the lactational amenorrhoea method to another method should occur. We acknowledged the limitations of using prevalence of modern contraceptive use as an outcome: there is potential for contraceptive uptake to be encouraged to achieve targets, without accounting for women's or couples' fertility intentions and their right to determine the timing and spacing of their children.

We followed WHO recommendations for medical eligibility to define modern contraceptives appropriate up to 12 months post partum. ${ }^{17}$ We categorised modern contraceptives as follows: long-acting and reversible contraceptives, including implants and intrauterine devices; short-acting contraceptives, including injectable, pills, emergency contraception, and male and female condoms, among others; permanent methods (male and female sterilisation); and lactational amenorrhoea. Contraceptives were further defined as "modern and appropriate" and "non-modern or non-appropriate". Non-modern were traditional methods, withdrawal, and abstinence. Non-appropriate were lactational ameno rrhoea if used after 6 months and calendar-based methods if used during the first 12 months post partum. We assumed that as most women breastfeed for up to 2 years, they would not fulfil the initiation requirement of having at least three regular menstrual cycles before 12 months.

\section{Statistical analysis}

The sample size was estimated on the basis of the following assumptions. Women in the intervention group wanting to limit or space their pregnancies would already use a modern contraceptive method at 6 months, when the lactational amenorrhoea method is no longer indicated. Therefore, although the main study outcome was prevalence of modern contraceptive use at 12 months post partum, we used country data at 6 months to set our sample size. The sample size was estimated with the 2010 Demographic and Health Survey data: ${ }^{5}$ we assumed a $5 \%$ modern contraceptive uptake in the control group (based on $7 \%$ of prevalence of modern contraceptive use among women using traditional or modern methods at 6 months post partum), and an increase to $20 \%$ in the experimental group (based on $15 \%$ of prevalence of modern contraceptive use among women in the general population, to which we added 5\% given the high unmet need for PPFP). Assuming an intracluster (health centre) correlation coefficient of 0.02 (no specific evidence existed for its value from the literature), ${ }^{18}$ the intervention group and the control group had each four study sites with at least 60 participants per site. This number allowed for a statistical power of $93 \%$ to detect a difference of $15 \%$ to a level of significance of $5 \%$. Assuming a $10 \%$ participant loss to follow up, each facility recruited at least 70 pregnant women. This equated to a cohort of at least 280 pregnant women in each study arm (four sites with 70 participants each), and in total at least 560 participants (appendix, pp 4-5). 
Data from all eligible participants were analysed with IBM SPSS Statistics (version 21.0), R (version 3.4.3), and WINPEPI (PEPI-for-Windows, version 11.50). Generalised linear mixed models (log binomial and log Poisson) were used to assess the effect of the package on prevalence ratios (PRs) of main outcomes with $95 \%$ CIs, comparing intervention and control groups while accounting for clustering and adjusting for potential confounders (women's baseline characteristics that were imbalanced between groups). Fisher's exact test was used for statistical significance of differences in outcomes between groups when samples were small, and models did not converge. WINPEPI was used to obtain a post-hoc global estimation of the intracluster correlation coefficient. We did a modified intention-to-treat analysis that included all women irrespective of whether they continued to visit the clinic after enrolment. Because women had to see a provider to receive the intervention package, we planned to do a per-protocol analysis focused on participants who attended all recommended follow-up visits. The study is registered with the Pan African Clinical Trials Registry (PACTR201609001784334).

\section{Role of the funding source}

The study funder had no role in study design, data collection, data analysis, data interpretation, or writing the Article. The corresponding author had full access to all the data in the study and had final responsibility for the decision to submit for publication.

\section{Results}

From July 27-Oct 17, 2016, 20 primary clinics were assessed for eligibility, where four clinics were randomly assigned to the intervention group and four to the control group (figure). Of 614 women asked to participate, 571 were enrolled in the study (286 received the intervention package, 285 received routine care), of whom 523 (260 in the intervention group, 263 in the control group) completed the 12 month study exit interview. Timely visits and missed visits between the intervention and control groups differed significantly at 6 weeks (181 [64\%] of 284 vs 96 [34\%] of 283) and 6 months (45 [16\%] of 280 vs 16 [6\%] of 283$)$. No participant in the intervention group attended all the recommended follow-up visits from clinic discharge to 12 months post partum, therefore the per protocol analysis was not done. The time from enrolment to exit interview spanned from July 27, 2016, to Dec 13, 2017.

Some baseline differences were noted between the intervention and control clusters (table 1), including length of exclusive breastfeeding of last child, interpregnancy intervals, ability to read among male partners, and usual travel time needed to get to the health centre.

At 12 months post partum, prevalence of modern contraceptive use in the intervention arm was about twice that of the control arm (55\% vs $29 \%$, adjusted prevalence ratio [PR] 1.79, 95\% CI 1.30-2.47); the ratio was similar

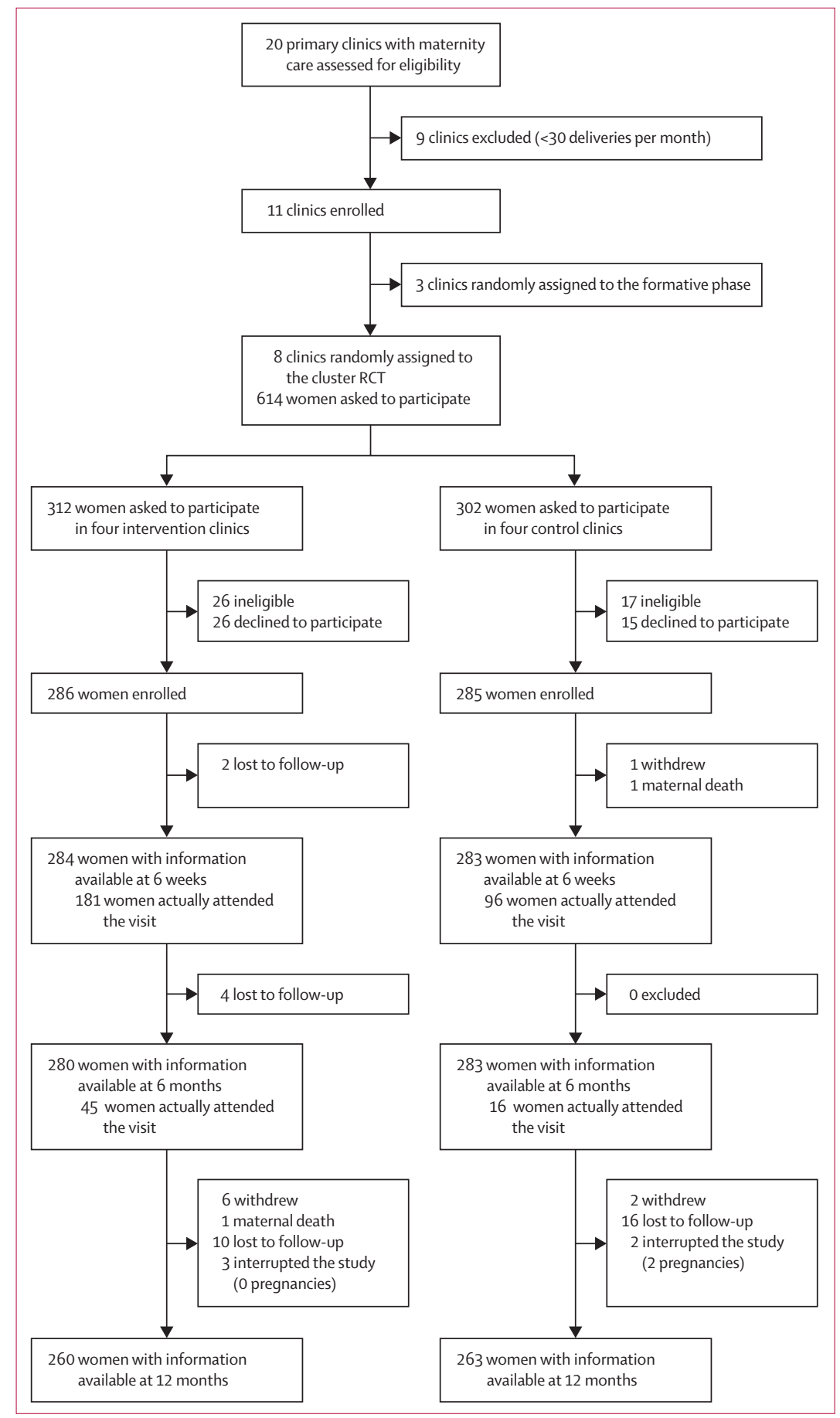

Figure: Trial profile

for both short-acting contraceptives or long-acting and reversible contraceptives (table 2). At 6 months, prevalence of modern contraceptive use (which included the lactational amenorrhoea method) in the intervention group was more than double that in the control group (59\% vs 24\%, adjusted PR 2.31, 1.44-3.71) and at 6 weeks post partum, prevalence of modern contraceptive use 


\begin{tabular}{|c|c|c|}
\hline & $\begin{array}{l}\text { Intervention } \\
(n=286)\end{array}$ & $\begin{array}{l}\text { Control } \\
(n=285)\end{array}$ \\
\hline \multicolumn{3}{|l|}{ General characteristics of women } \\
\hline Maternal age (years) & $27(16-47)$ & $24(15-44)$ \\
\hline \multicolumn{3}{|l|}{ Marital status } \\
\hline Married or in a relationship & $282(99 \%)$ & $280(98 \%)$ \\
\hline $\begin{array}{l}\text { Single, widow, separated, or } \\
\text { divorced }\end{array}$ & $4(1 \%)$ & $5(2 \%)$ \\
\hline \multicolumn{3}{|l|}{ Education level } \\
\hline Never attended school & $202(70 \%)$ & $205(72 \%)$ \\
\hline Primary school & $41(14 \%)$ & $40(14 \%)$ \\
\hline Secondary school & $43(15 \%)$ & $40(14 \%)$ \\
\hline Beyond secondary & $0(0 \%)$ & $0(0 \%)$ \\
\hline Able to read an official language & $78(27 \%)$ & $91(32 \%)$ \\
\hline \multicolumn{3}{|l|}{ Living setting } \\
\hline Rural or urban-rural & $216(76 \%)$ & $226(80 \%)$ \\
\hline Urban & $70(25 \%)$ & $59(21 \%)$ \\
\hline \multicolumn{3}{|l|}{ Usual travel time to health centre } \\
\hline$<30 \mathrm{~min}$ & $112 / 269(42 \%)$ & $77 / 275(28 \%)$ \\
\hline $30-60 \mathrm{~min}$ & $105 / 269(39 \%)$ & 133/275 (48\%) \\
\hline$>1 \mathrm{~h}$ & $52 / 269(19 \%)$ & $65 / 275(24 \%)$ \\
\hline \multicolumn{3}{|l|}{ Employment } \\
\hline Housewife or farmer & $230(80 \%)$ & $233(82 \%)$ \\
\hline Student & $25(9 \%)$ & $21(7 \%)$ \\
\hline Business & $19(7 \%)$ & $21(7 \%)$ \\
\hline Other & $12(4 \%)$ & $10(4 \%)$ \\
\hline \multicolumn{3}{|l|}{ Maternity and contraception } \\
\hline \multicolumn{3}{|l|}{ Previous pregnancies } \\
\hline Yes & $238 / 283(84 \%)$ & $228 / 285(80 \%)$ \\
\hline Median number & $3(0-8)$ & $2(0-8)$ \\
\hline \multicolumn{3}{|l|}{ Pregnancy outcomes } \\
\hline Livebirth & $3(0-9)$ & $3(0-8)$ \\
\hline Stillbirth & $0(0-2)$ & $0(0-3)$ \\
\hline Abortion & $0(0-2)$ & $0(0-3)$ \\
\hline Extra-uterine & $0(0)$ & $0(0-2)$ \\
\hline \multicolumn{3}{|l|}{ Living biological children } \\
\hline Yes & $231 / 238(97 \%)$ & $220 / 228(97 \%)$ \\
\hline Median number & $3(0-7)$ & $2(0-7)$ \\
\hline \multicolumn{3}{|l|}{ Age of last child } \\
\hline$\geq 2$ years & 218/231(94\%) & $211 / 220(96 \%)$ \\
\hline Breastfeeding of last child & $232 / 234(99 \%)$ & 224/227 (99\%) \\
\hline \multicolumn{3}{|c|}{ Length of exclusive breastfeeding (months) } \\
\hline$<1$ & $138 / 232(60 \%)$ & $144 / 224(64 \%)$ \\
\hline $1-5$ & 19/232 (8\%) & $41 / 224(18 \%)$ \\
\hline$\geq 6$ & 75/232 (32\%) & 39/224 (17\%) \\
\hline \multicolumn{3}{|l|}{ Interpregnancy interval (years) } \\
\hline$\geq 2$ & 197/237 (83\%) & $153 / 228(67 \%)$ \\
\hline \multirow[t]{2}{*}{$<2$} & 40/237 (17\%) & 75/228 (33\%) \\
\hline & \multicolumn{2}{|c|}{ (Table 1 continues in next column) } \\
\hline
\end{tabular}

(including lactational amenorrhoea) in the intervention group was four times that in the control arm (42\% vs 10\%, adjusted PR $3 \cdot 88,1 \cdot 46-10 \cdot 35)$. No women used the lactational amenorrhoea method in the control group versus a significantly higher proportion of about one in

\begin{tabular}{|c|c|c|}
\hline & $\begin{array}{l}\text { Intervention } \\
(n=286)\end{array}$ & $\begin{array}{l}\text { Control } \\
(n=285)\end{array}$ \\
\hline \multicolumn{3}{|l|}{ (Continued from previous column) } \\
\hline Current pregnancy planned & $143(50 \%)$ & $160(56 \%)$ \\
\hline $\begin{array}{l}\text { Contraception used during year } \\
\text { before pregnancy }\end{array}$ & $82(29 \%)$ & $77(27 \%)$ \\
\hline \multicolumn{3}{|l|}{ Main reason for non-use } \\
\hline Did not know about methods & $117 / 204(57 \%)$ & $41 / 208(20 \%)$ \\
\hline Pregnancy desire & $45 / 204(22 \%)$ & $49 / 208(24 \%)$ \\
\hline Partner or family opposition & $12 / 204(6 \%)$ & $29 / 208(14 \%)$ \\
\hline Fear of side-effects & $9 / 204(4 \%)$ & $25 / 208(12 \%)$ \\
\hline Other & $21 / 204(10 \%)$ & $64 / 208(31 \%)$ \\
\hline \multicolumn{3}{|l|}{ Main contraceptive used } \\
\hline Injectable & $42 / 82(51 \%)$ & $48 / 77(63 \%)$ \\
\hline Pill & $19 / 82(23 \%)$ & $15 / 77(20 \%)$ \\
\hline Implant & $17 / 82(21 \%)$ & $13 / 77(17 \%)$ \\
\hline Male condom & $3 / 82(4 \%)$ & $1 / 77(1 \%)$ \\
\hline Standard day method & $1 / 82(1 \%)$ & $0(0 \%)$ \\
\hline \multicolumn{3}{|c|}{ General characteristics of male partners* } \\
\hline Median age (years) $\dagger$ & $33(19-77)$ & $31(17-70)$ \\
\hline \multicolumn{3}{|l|}{ Education level } \\
\hline Never attended school & $189 / 282(67 \%)$ & $179 / 280(64 \%)$ \\
\hline Primary school & $53 / 282(19 \%)$ & $63 / 280(23 \%)$ \\
\hline Secondary school & $35 / 282(12 \%)$ & $37 / 280(13 \%)$ \\
\hline Beyond secondary & $5 / 282(2 \%)$ & $1 / 280(0 \%)$ \\
\hline Able to read an official language & $91 / 281(32 \%)$ & $145 / 279(52 \%)$ \\
\hline \multicolumn{3}{|l|}{ Employment } \\
\hline Farmer & $174 / 281(62 \%)$ & $204 / 280(73 \%)$ \\
\hline Business & 32/281 (11\%) & $20 / 280(7 \%)$ \\
\hline Craftsman & $21 / 281(8 \%)$ & $7 / 280(3 \%)$ \\
\hline Other & $54 / 281(19 \%)$ & 49/280 (18\%) \\
\hline \multicolumn{3}{|c|}{$\begin{array}{l}\text { Data are } n / \mathrm{N}(\%) \text { or median (range). * Information collected from the enrolled } \\
\text { women about their partners; male partners were not interviewed directly. } \mathrm{nn}=61 \\
\text { in the intervention group; } \mathrm{n}=80 \text { in the control group. }\end{array}$} \\
\hline
\end{tabular}

ten women in the intervention group at both 6 weeks and 6 months post partum (table 2).

In terms of the mix between methods, within each of the trial arms, the distribution between short-acting contraceptives and long-acting and reversible contraceptive users was approximatively even, with slightly more short-acting contraceptive users at 6 weeks and 6 months, and slightly more long-acting and reversible contraceptives users at 12 months (table 2). Implant was by far the preferred method among long-acting and reversible contraceptives users (appendix). There was no male or female sterilisation. The post-hoc global estimation of the intracluster correlation coefficient was $0.036(0.064$ for the intervention group and 0.009 for the control group).

\section{Discussion}

Overall, our results showed that the implementation of a package of six low-technology PPFP interventions was an effective strategy to increase the use of modern 
contraceptives for up to 12 months post partum in women in a predominantly rural region of Burkina Faso.

In both arms, the prevalence of modern contraceptive use increased from 6 weeks to 6 months and remained relatively steady until 12 months. The prevalence of modern contraceptive use among participants in the control arm at 6 months was at a level similar to that of the projected prevalence for 2017 among married women in Burkina Faso (25\%). ${ }^{19}$ In comparison, the prevalence among participants who received the package of interventions $(23 \cdot 4 \%$ without the lactational amenorrhoea method) had reached the national prevalence among married women by 6 weeks, before increasing to more than twice the national prevalence at 6 months and 12 months.

The modern contraceptive method mix at 12 months within both study arms reflected that reported in the general population in Burkina Faso from 2014 to 2015 (ie, $40 \%$ implants, $35 \%$ injectables, $13 \%$ pills, $8 \%$ male condoms, $3 \%$ intrauterine device, and other methods). ${ }^{20}$

Our low-dose, high-frequency approach required women to attend the routinely recommended followup visits for timely adoption of contraceptives. Client counselling, supported by the PPFP tool, combined with the provision of appointment cards, might have contributed to the marked differences in timely visits between women in the intervention and control groups at 6 weeks and 6 months, although the proportion reduced in both groups over time and no women attended all the follow-up visits.

As for the use of the lactational amenorrhoea method at 6 weeks and 6 months post partum, although there was a significant difference between the intervention and control arms, our results contrast with the 2010 Demographic and Health Survey findings that showed that $25 \%$ of infants younger than 6 months were exclusively breastfed. ${ }^{5}$ Early supplementation rooted in customary practices are reported to be common ${ }^{21}$ and might explain our results as well as the stagnant median duration of exclusive breastfeeding in Burkina Faso (0.4 month in 1993 and 0.6 month in 2010), which reflects the situation in other low-income countries. ${ }^{22}$ Also according to the 2010 Demographic and Health Survey, the median duration of amenorrhoea was 12 months and suggests that breastfeeding, although not exclusive, must be frequent enough to induce amenorrhoea. Therefore, our intervention package covered lactational amenorrhoea benefits, requirements, and limitations in the PPFP counselling tool along with information on other effective contraceptive options. Our package did not include a targeted promotion of the lactational amenorrhoea method by health staff because it was not perceived as an efficient use of limited resources, ${ }^{15}$ although studies in sub-Saharan Africa showed that such promotion can increase the prevalence of exclusive breastfeeding (eg, through peer counsellors ${ }^{23}$ or the Ten Steps to Successful Breastfeeding ${ }^{24}$ ). In fact,

\begin{tabular}{|c|c|c|c|}
\hline & Intervention & Control & $\begin{array}{l}\text { Adjusted prevalence } \\
\text { ratio }(95 \% \mathrm{Cl})^{*}\end{array}$ \\
\hline \multicolumn{4}{|l|}{12 months (primary outcome) } \\
\hline Modern and appropriate methods & $143 / 260(55 \%)$ & $76 / 263(29 \%)$ & $1 \cdot 79(1 \cdot 30-2 \cdot 47)$ \\
\hline Long-acting or permanent methods & $76 / 260(30 \%)$ & $44 / 263(17 \%)$ & $1.66(1 \cdot 17-2 \cdot 35)$ \\
\hline Short-acting methods & $67 / 260(26 \%)$ & $32 / 263(12 \%)$ & $2 \cdot 01(1.18-3 \cdot 43)$ \\
\hline Non-modern or non-appropriate methods & $1 / 260(0 \%)$ & $0 / 263(0 \%)$ & .. \\
\hline No method & $116 / 260(45 \%)$ & $187 / 263(71 \%)$ & $0.65(0.51-0.83)$ \\
\hline \multicolumn{4}{|l|}{6 weeks } \\
\hline Modern and appropriate methods & $120 / 284(42 \%)$ & $29 / 283(10 \%)$ & $3 \cdot 88(1 \cdot 46-10 \cdot 35)$ \\
\hline Long-acting or permanent methods & $32 / 284(11 \%)$ & $13 / 283(5 \%)$ & $2.3(0.95-5.58)$ \\
\hline Short-acting methods & $38 / 284(13 \%)$ & $16 / 283(6 \%)$ & $2 \cdot 45(1.34-4.49)$ \\
\hline Lactational amenorrhea method & $50 / 284(18 \%)$ & $0 / 283(0 \%)$ &..$\dagger$ \\
\hline Non-modern or non-appropriate methods & $0 / 284(0 \%)$ & $0 / 283(0 \%)$ & .. \\
\hline No method & $164 / 284(58 \%)$ & $254 / 283(90 \%)$ & $0.51(0.21-1 \cdot 21)$ \\
\hline \multicolumn{4}{|l|}{6 months } \\
\hline Modern and appropriate methods & $164 / 280(59 \%)$ & $68 / 283(24 \%)$ & $2 \cdot 31(1 \cdot 44-3 \cdot 71)$ \\
\hline Long-acting or permanent methods & $59 / 280(21 \%)$ & $33 / 283(12 \%)$ & $1.65(1.09-2.48)$ \\
\hline Short-acting methods & $70 / 280(25 \%)$ & $35 / 283(12 \%)$ & $2 \cdot 05(1 \cdot 35-3 \cdot 12)$ \\
\hline Lactational amenorrhea method & $35 / 280(13 \%)$ & $0 / 283(0 \%)$ &.$+\dagger$ \\
\hline Non-modern or non-appropriate methods & $0 / 280(0 \%)$ & $0 / 283(0 \%)$ &.. \\
\hline No method & $116 / 280(41 \%)$ & $215 / 283(76 \%)$ & $0.49(0.26-0.92)$ \\
\hline \multicolumn{4}{|c|}{$\begin{array}{l}\text { Data are } \mathrm{n} / \mathrm{N}(\%) \text {, unless indicated differently. Point and interval estimations were obtained using log binomial or log } \\
\text { Poisson models for prevalence ratios. *Accounting for clustering and adjusted for usual travel time to health centre } \\
\text { and ability to read an official language (among partners). †Fisher's exact test, two-sided p value }<0.001 \text {. }\end{array}$} \\
\hline
\end{tabular}

most lactational amenorrhoea method users came from a single intervention study site (Bouré), where the main service provider was found to be deeply convinced by the benefits of the lactational amenorrhoea method and counselled on it.

To our knowledge, Yam Daabo is the first study using a PPFP package approach in Africa. Its results add to the body of evidence regarding the effectiveness of various packages of different interventions on postpartum contraceptive use, which is relatively sparse. The package strategy has previously been shown to result in earlier and increased use of contraception by post-partum women in two studies with preintervention and postintervention designs that were implemented in 2002 in Guatemala ${ }^{25}$ and in 1998 in Russia. ${ }^{26}$ They tested a comprehensive package of maternal and child health interventions, including PPFP, in the postnatal period. In Guatemala, the interventions took place at primary and secondary levels of care and comprised information education and counselling materials, training of providers, and monitoring at the facility and community levels; the proportion of women using a modern contraceptive three months after birth increased from $18 \%$ to $25 \%$. In Russia, the package comprised the provision of supplies, training providers, and quality of care improvement in a women's hospital; before being discharged home, $65 \%$ of post-partum women adopted a modern contraceptive method following the intervention 
as compared to $0 \%$ before. Neither of the two studies had a longer follow-up to assess change in contraceptive use. In contrast to theses studies, which did not directly focus on PPFP, and to studies investigating specific PPFP methods or approaches (with most of them assessing modern contraceptive prevalence at 6 months or less), our research focused on PPFP within an overall approach of strengthening routine antenatal care and postnatal care services. Moreover, modern contraceptive prevalence in our trial was assessed up to 12 months after childbirth. Our study also stands out as it did not investigate a specific programmatic innovation or the promotion of a particular PPFP method: fertility and contraceptive choices were up to the women and couples, and concentrated on primary health-care centres, given the growing availability of antenatal care, delivery, and postnatal care services at primary care level in lowresource settings, including rural areas. This offers enhanced client-provider contact points to minimise missed health services opportunities to address the contraceptive needs of postpartum women and couples. Our study also contributes to a global (a posteriori) estimation of the intracluster correlation coefficient, which was about 0.03 , somewhat larger than the initially-assumed value of 0.02 (under this assumption of 0.03 , the power is above $80 \%$ ).

Our trial has several limitations. First, despite being randomised, the study included a total of eight clusters, which was sufficient according to our statistical hypotheses but is relatively modest and therefore at increased risk of groups not being fully similar. However, when controlled for differences in baseline characteristics, the results did not change substantially (the unadjusted PR at 12 months was 1.90). Second, the nature of this pragmatic facility-based trial did not allow blinding of participants, providers, and interviewers, and we cannot rule out assessment bias nor a Hawthorn effect. Third, although we used independent interviewers, we also cannot exclude an enhanced effect size due to a bias towards socially desirable answers especially in this facility-based research. Fourth, the paired urban study sites were both in Yako and we cannot exclude intervention contamination. However, a Hawthorn effect, information and assessment bias, and contamination alone cannot contribute to the large contraceptive use reported at different measurement points. Fifth, policy makers and programme managers might wish to implement a single most effective intervention. Our trial design does not allow one to distinguish which intervention in the package had the greatest effect on contraceptive use, for which further research would be required. However, interventions were chosen based on multiple stakeholder perspectives and integrated into the package because they were perceived to be of low-technology, adapted to resourcelimited settings, while strengthening existing services and therefore easily feasible and replicable. Sixth, women using modern contraceptives at 12 months would require follow-up until 2 years and beyond to study their patterns of contraceptive use and assess whether they continue to be more successful in spacing or limiting pregnancies according to their reproductive choices.

The implementation of a package of six low-technology PPFP interventions designed in a participatory manner to strengthen the continuum of antenatal care and postnatal care services in every day care in primary health clinics significantly raised the proportion of women using the lactational amenorrhoea method up to 6 months post partum and short-acting and long-acting reversible modern contraceptives up to 12 months post partum. The large-scale implementation of a similar package in similar settings could contribute to decreasing the unmet need for post partum family planning among women and couples and increasing the likelihood of meeting FP2020 goals.

\section{Contributors}

NTT, SK, AS, MEG, and SL designed the study protocol and instruments. AC, BT, TM, FS, WMEY, SZ, and SK contributed to the final study protocol, study instruments, and led the field implementation. NTT, SL, SZ, AC-K, JK and MEG monitored the study implementation. AS, SL, FS $\mathrm{AC}$, and NTT contributed to data management and analysis. AC-K coordinated the project between Burkina Faso and WHO. NTT wrote the first draft of the manuscript with the contributions of MEG, AC-K, JK, AS, SL, SK, BT, AC, TM, WMEY, FS, and SZ. All authors contributed toward data analysis, drafting, and revising the paper and agreed to be accountable for all aspects of the work.

\section{Declaration of interests}

We declare no competing interests.

\section{Data sharing}

Requests for the anonymised, coded trial data can be made to the Department of Reproductive Health and Research, World Health Organization (reproductivehealth@who.int). Data sharing is subject to WHO data sharing policies and data use agreements with the participating research centres.

\section{Acknowledgments}

We thank all the participants, staff in the health centres, and research assistants in the study sites and in Ouagadougou. The Government of France generously provided funding for this research grant in the context of the Muskoka Initiative on Maternal and Child Health.

\section{References}

1 Cleland J, Bernstein S, Ezeh A, Faundes A, Glasier A, Innis J. Family planning: the unfinished agenda. Lancet 2006; 368: 1810-27.

2 World Health Organization. Programming strategies for postpartum family planning. Geneva, 2013.

3 Moore Z, Pfitzer A, Gubin R, Charurat E, Elliott L, Croft T. Missed opportunities for family planning: an analysis of pregnancy risk and contraceptive method use among postpartum women in 21 low- and middle-income countries. Contraception 2015; 92: 31-39.

4 Rossier C, Bradley SE, Ross J, Winfrey W. Reassessing unmet need for family planning in the postpartum period. Stud Fam Plann 2015 46: 355-67.

5 Institut National de la Statistique et de la Démographie (INSD) et ICF International. Enquête Démographique et de Santé et à Indicateurs Multiples du Burkina Faso 2010. Calverton: INSD et ICF International, 2012.

6 Borda M, Winfrey W. Postpartum fertility and contraception: an analysis of findings from 17 countries. Baltimore: Jhpiego, 2010

7 WHO. Trends in maternal mortality: 1990-2015: estimates from WHO, UNICEF, UNFPA, World Bank Group, and the United Nations Population Division: executive summary. Number WHO/RHR/15.23. Geneva: World Health Organization, 2015. 
8 Ross JA, Winfrey WL. Contraceptive use, intention to use and unmet need during the extended postpartum period. Inter Fam Plan Perspect 2001; 27: 20-27.

9 Adeyemi AB, Ijadunola KT, Orji EO, Kuti O, Alabi MM. The unmet need for contraception among Nigerian women in the first year post-partum. Eur J Contracept Reprod Health Care 2005; 10: 229-34.

10 Sonalkar S, Mody S, Gaffield ME. Outreach and integration programs to promote family planning in the extended postpartum period. Int J Gynecol Obstet 2014; 124: 193-97.

11 Cleland J, Shah IH, Daniele M. Interventions to improve postpartum family planning in low- and middle-income countries: program implications and research priorities. Stud Fam Plann 2015; 46: 423-41.

12 Blazer C, Prata N. Postpartum family planning: current evidence on successful interventions. Open Access J Contracept 2016; 2016: 53.

13 Tran NT, Gaffield ME, Seuc A, et al. Effectiveness of a package of postpartum family planning interventions on the uptake of contraceptive methods until twelve months postpartum in Burkina Faso and the Democratic Republic of Congo: the YAM DAABO study protocol. BMC Health Serv Res 2018; 18: 439.

14 Sonalkar S, Mody S, Gaffield ME. Outreach and integration programs to promote family planning in the extended postpartum period. Int J Gynecol Obstet 2014, 124: 193-97.

15 Tran NT, Yamaego WME, Langwana F, et al. Participatory action research to identify a package of interventions to promote postpartum family planning in Burkina Faso and the Democratic Republic of Congo. BMC Women's Health 2018; 18: 122.

16 Tran NT, Yamaego M, Langwana F, et al. Birth spacing and informed family planning choices after childbirth in Burkina Faso and the Democratic Republic of Congo: participatory action research to design and evaluate a decision-making tool for providers and their clients. Patient Educ Couns 2018; 101: 1871-75.

17 WHO. Medical eligibility criteria for contraceptive use. Geneva: World Health Organization, 2015.
18 Murray DM, Varnell SP, Blitstein JL. Design and analysis of group-randomized trials: a review of recent methodological developments. Am J Public Health 2004; 94: 423-32.

19 Cahill N, Sonneveldt E, Stover J, et al. Modern contraceptive use, unmet need, and demand satisfied among women of reproductive age who are married or in a union in the focus countries of the Family Planning 2020 initiative: a systematic analysis using the Family Planning Estimation Tool. Lancet 2018; 391: 870-82.

20 Zimmerman L, Olson H, Group PPI, Tsui A, Radloff S. PMA2020: rapid turn-around survey data to monitor family planning service and practice in ten countries. Stud Fam Plann 2017; 48: 293-303.

21 Cresswell JA, Ganaba R, Sarrassat S, et al. Predictors of exclusive breastfeeding and consumption of soft, semi-solid or solid food among infants in Boucle du Mouhoun, Burkina Faso: a cross-sectional survey. PLoS One 2017; 12: e0179593.

22 Fabic MS, Choi Y. Assessing the quality of data regarding use of the lactational amenorrhea method. Stud Fam Plann 2013; 44: 205-21.

23 Tylleskär T, Jackson D, Meda N, et al. Exclusive breastfeeding promotion by peer counsellors in sub-Saharan Africa (PROMISE-EBF): a cluster-randomised trial. Lancet 2011; 378: 420-27.

24 Yotebieng M, Labbok M, Soeters HM, et al. Ten Steps to Successful Breastfeeding programme to promote early initiation and exclusive breastfeeding in DR Congo: a cluster-randomised controlled trial. Lancet Glob Health 2015; 3: e546-55.

25 Jacobs E, Brambila C, Vernon R. Reproductive health care in the postnatal period in Guatemala. Washington, DC: Frontiers in Reproductive Health, Population Council, 2002.

26 Stephenson P, Frolova F, Donnay O, Melnick T, Worzala C. Improving women's health services in the Russian Federation: results of a pilot project. Washington, DC: World Bank, 1998. 